\title{
Tronchuda cabbage flavonoids uptake by Pieris brassicae
}

\author{
Federico Ferreres ${ }^{\text {a }}$, Carla Sousa ${ }^{\mathrm{b}}$, Patrícia Valentão ${ }^{\mathrm{b}}$, José A. Pereira ${ }^{\mathrm{c}}$, \\ Rosa M. Seabra ${ }^{b}$, Paula B. Andrade ${ }^{b, *}$ \\ ${ }^{a}$ Research Group on Quality, Safety and Bioactivity of Plant Foods, Department of Food Science and Technology, CEBAS (CSIC), \\ P.O. Box 164, 30100 Campus University, Espinardo, Murcia, Spain \\ ${ }^{\mathrm{b}}$ REQUIMTE/Serviço de Farmacognosia, Faculdade de Farmácia, Universidade do Porto, R. Aníbal Cunha, 164, 4050-047 Porto, Portugal \\ ${ }^{\mathrm{c}}$ CIMOIESAB, Quinta de Sta Apolónia, Apartado 1172, 5301-855 Bragança, Portugal
}

Received 31 July 2006; received in revised form 3 October 2006

Available online 30 November 2006

\begin{abstract}
The flavonoid pattern of larvae of cabbage white butterfly (Pieris brassicae L.; Lepidoptera: Pieridae) reared on the leaves of tronchuda cabbage was analysed by HPLC-DAD-MS/MS-ESI. Twenty flavonoids were identified or characterised, namely 16 kaempferol and four quercetin derivatives. Kaempferol 3- $O$-sophoroside, a minor component of tronchuda cabbage, was found to be the main component in $P$. brassicae (15.8\%). Apart from this, only two other flavonoids present in significant amounts in tronchuda cabbage (kaempferol 3- $O$-sophoroside-7- $O$-glucoside and kaempferol 3- $O$-sophoroside-7- $O$-sophoroside) were found in the larvae. The larvae have high amounts of quercetin derivatives $(18.5 \%)$, which were present only in trace amounts in tronchuda cabbage extracts, suggesting that $P$. brassicae is able to selectively sequester these flavonoids. The occurrence of a high content of flavonoids not detectable in tronchuda cabbage extracts indicates that $P$. brassicae larvae are able to metabolize dietary flavonoids.
\end{abstract}

(C) 2006 Elsevier Ltd. All rights reserved.

Keywords: Pieris brassicae L. larvae; Tronchuda cabbage; Brassica oleracea L. var. costata DC; Flavonoids

\section{Introduction}

Larvae of Pieris brassicae L. (Lepidoptera: Pieridae) are specialists on crucifers, whereas adults feed of the nectar of a variety of plants. The larvae can feed on various species of Brassicaceae, namely, cauliflower, cabbage, turnip, nasturtium and, more rarely, on red cabbage and radish.

The close association between Pieris sp. butterflies and crucifers was linked to the presence of glucosinolates in these plants (Renwick, 2002). As well as glucosinolates, flavonoids can modulate the feeding behaviour of larvae and oviposition of adult insects (van Loon et al., 2002).

Flavonoid uptake is relatively widespread in the Lepidoptera, in particular in butterfly families like the Papilionidae, Nymphalidae, and Lycaenidae, where they form part of the wing pigmentation (Burghardt et al., 1997, 2001;

\footnotetext{
${ }^{*}$ Corresponding author. Tel.: +351 222078935; fax: +351 222003977.

E-mail address: pandrade@ff.up.pt (P.B. Andrade).
}

Schittko et al., 1999). In fact, although most of pigments are synthesised de novo during scale development in the pupa, some are secondary plant metabolites taken up from the larval diet since insects are unable to synthesize flavonoids or their precursors de novo (Knüttel and Fiedler, 2001). Feeding experiments proved the dietary origin of the flavonoids (Burghardt et al., 1997; Schittko et al., 1999; Knüttel and Fiedler, 2001; Harborne and Grayer, 1994). Thus, flavonoid uptake and metabolism by insects is strongly dependent on the specific flavonoid pattern of their host plants (Burghardt et al., 1997, 2001; Schittko et al., 1999; Geuder et al., 1997).

The flavonoids sequestered by the larvae are subsequently metabolised, stored and transferred into the wings during the late pupal stage (Geuder et al., 1997). Flavonoids are known for their antioxidant potential (Ferreres et al., 2006; Vrchovská et al., 2006) and perhaps they act as antibiotic and antiviral agents in insects (Harborne and Grayer, 1994). 
In what concerns flavonoids patterns it was shown that (i) larvae sequester only specific fractions of the flavonoid load of the host plant, (ii) the sequestered flavonoids are subjected to various glycosydation processes, (iii) the flavonoid content of butterflies from the same species may drastically vary according to the host plant actually used during the larval stages, and (iv) female butterflies tend to be richer in flavonoids than males (Burghardt et al., 2001).

As referred above, flavonoids in the insects are positively associated with the amounts of flavonoids in the food that they had consumed (Burghardt et al., 2000). Larvae selectively sequester and metabolise quercetin and kaempferol derivatives, the predominant flavonoids in the analysed plants. Other flavonoids such as myricetin derivatives, flavones and isoflavonoids were mostly excreted (Burghardt et al., 2001).

As far as we know, there is no study concerning the sequestration of phenolic compounds by $P$. brassicae from tronchuda cabbage leaves (Brassica oleracea L. var. costata DC) or other cabbages. This study can be relevant from the nutritional point of view, considering that the larvae may accumulate or even metabolize trochuda cabbage constituents, namely complex flavonol glycosides (Ferreres et al., 2005 , 2006), constituting a source of potential bioactive compounds not available in nature.

In this paper, the flavonoids present in larvae of $P$. brassicae were tentatively identified by HPLC-DADMS/MS-ESI and they were compared to the flavonoid pattern of the tronchuda cabbage external leaves, which were the only ones on which they have fed. Some considerations about ingestion, metabolism and accumulation of flavonoids in $P$. brassicae are made.

\section{Results and discussion}

\subsection{Characterisation of P. brassicae phenolic compounds}

The HPLC-DAD-MS/MS-ESI screening of the aqueous methanolic extract of $P$. brassicae larvae (Fig. 1) shows several peaks with UV spectra characteristic of flavonols, with two maxima at around 260 and $350 \mathrm{~nm}$ (Table 1), and their corresponding acylated derivatives (Table 2). The UV spectra shape of the acylated flavonols resembles the overlapping of a flavonol spectrum with a hydroxycinnamic acid one, with a broad maximum around $310-330 \mathrm{~nm}$ and a short maximum or shoulder around $265-271 \mathrm{~nm}$ (Table 2 ), which, therefore, can be misunderstood as a cinnamic acid derivative. Some spectra are poorly defined because the compounds are found in trace amounts and/or coelute with other ones. Thus, UV spectra of compounds 7, 10 and $\mathbf{1 2}$ are not included in Table 2, and those of compounds $\mathbf{8}$, $\mathbf{1 3}$ and $\mathbf{1 6}$ differ from the expected for isolated and purified compounds, probably due to possible co-elution with other products.

The ESI-MS/MS ion trap study confirms the above mentioned and shows several flavonoids which are structurally similar to those already described in various Brassicaceae species (Ferreres et al., 2005, 2006; Llorach et al., 2003; Vallejo et al., 2004). These compounds are characterised to be

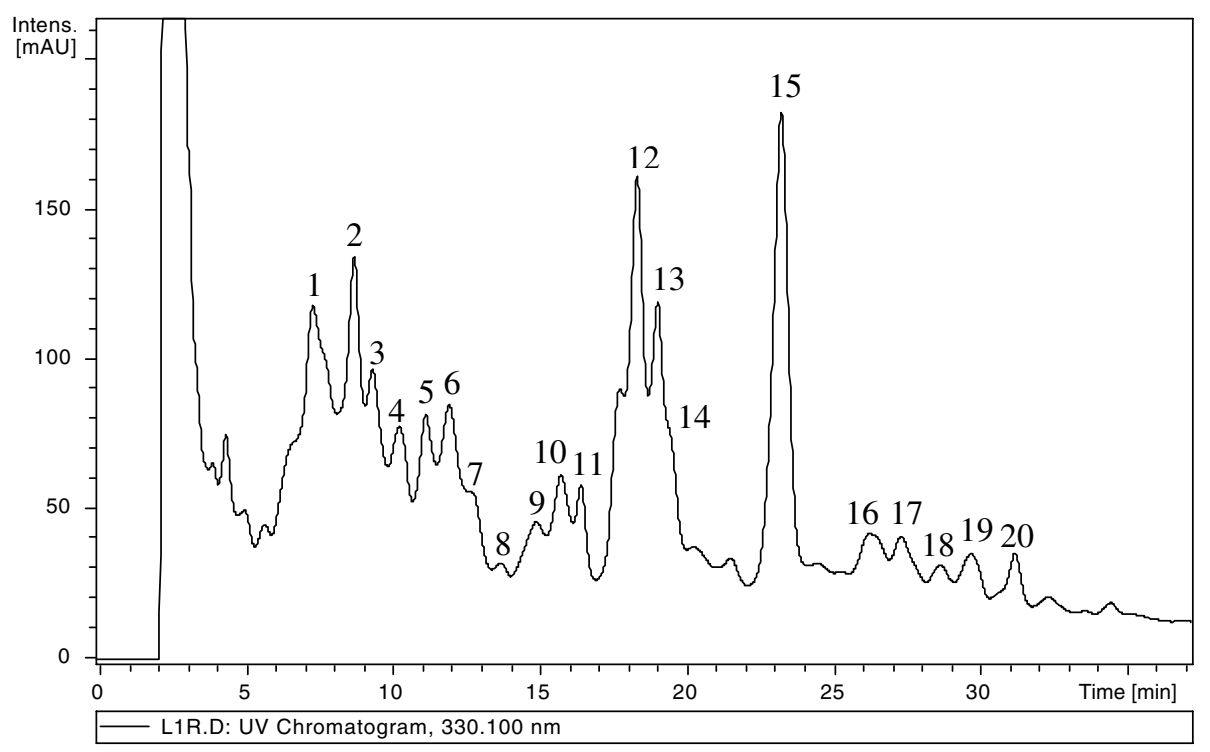

Fig. 1. HPLC-DAD phenolic profile of larvae hydromethanolic extract. Detection at $330 \mathrm{~nm}$. Peaks: (1) quercetin 3-O-sophoroside-7-O-glucoside; (2) kaempferol 3- $O$-sophoroside-7- $O$-glucoside; (3) kaempferol 3- $O$-sophoroside-7- $O$-sophoroside; (4) quercetin 3- $O$-(feruloyl)-triglucoside-7- $O$-glucoside; (5) kaempferol 3-O-(sinapoyl)-triglucoside-7-O-glucoside; (6) kaempferol 3-O-(feruloyl)-triglucoside-7-O-glucoside; (7) kaempferol 3- $O$-( $p$-coumaroyl)triglucoside-7- $O$-glucoside; (8) kaempferol 3-O-(methoxycaffeoyl)-sophoroside-7- $O$-glucoside; (9) kaempferol 3- $O$-(caffeoyl)-sophoroside-7- $O$-glucoside; (10) quercetin 3- $O$-( $p$-coumaroyl)-sophoroside; (11) kaempferol 3- $O$-( $p$-coumaroyl)-triglucoside; (12) kaempferol 3- $O$-( $p$-coumaroyl)-sophoroside; (13) kaempferol 3-O-(methoxycaffeoyl)-sophoroside; (14) quercetin 3- $O$-sophoroside; (15) kaempferol 3-O-sophoroside; (16) kaempferol 3- $O$-( $p$-coumaroyl)sophoroside (isomer); (17) kaempferol 3-O-(disinapoyl)-triglucoside-7-O-glucoside; (18) kaempferol 3-O-(feruloyl/sinapoyl)-triglucoside-7- $O$-glucoside; (19) quercetin 3-O-(feruloyl)-triglucoside; (20) kaempferol 3- $O$-glucoside. 
Table 1

$R_{\mathrm{t}}$, UV, -MS: $[\mathrm{M}-\mathrm{H}]^{-},-\mathrm{MS}^{2}[\mathrm{M}-\mathrm{H}]^{-}$and $-\mathrm{MS}^{3}[(\mathrm{M}-\mathrm{H}) \rightarrow 625 / 609]^{-}$data of flavonol glycosides without acylation found in $P$. brassicae ${ }^{\mathrm{a}}$

\begin{tabular}{|c|c|c|c|c|c|c|c|c|c|}
\hline \multirow[t]{2}{*}{ Compounds ${ }^{\mathrm{b}}$} & \multirow[t]{2}{*}{$R_{\mathrm{t}}(\min )$} & \multirow[t]{2}{*}{ UV (nm) } & \multirow{2}{*}{$\begin{array}{l}{[\mathrm{M}-\mathrm{H}]^{-}} \\
(\mathrm{m} / \mathrm{z})\end{array}$} & \multicolumn{3}{|c|}{$-\mathrm{MS}^{2}[\mathrm{M}-\mathrm{H}]^{-}(m / z)(\%)$} & \multicolumn{3}{|c|}{$-\mathrm{MS}^{3}[(\mathrm{M}-\mathrm{H}) \rightarrow 625 / 609]^{-}(\mathrm{m} / \mathrm{z})(\%)$} \\
\hline & & & & -162 & -180 & -324 & -162 & -180 & -324 \\
\hline 1 Querc-3-Soph-7-Glc ${ }^{c}$ & 7.2 & & 787 & $625(100)$ & & & $463(25)$ & $445(50)$ & $300(100)$ \\
\hline 3 Kaempf-3-Soph-7-Soph ${ }^{\mathrm{c}}$ & 9.2 & & 933 & $771(25)$ & & $609(100)$ & & $429(45)$ & $285(100)$ \\
\hline 14 Querc-3-Soph & 19.4 & $255,267,350$ & 625 & $463(15)$ & $445(25)$ & $300(100)$ & & & \\
\hline 15 Kaempf-3-Soph & 23.1 & $265,293 \mathrm{sh}, 347$ & 609 & 447 (10) & $529(50)$ & $285(100)$ & & & \\
\hline 20 Kaempf-3-Glc & 31.0 & $265,295 \mathrm{sh}, 348$ & 447 & $285(100)$ & & & & & \\
\hline
\end{tabular}

${ }^{a}$ Main observed fragments. Other ions were found but they have not been included.

${ }^{\text {b }}$ Querc, quercetin; Kaempf, kaempferol; Soph, sophoroside; Glc, glucoside.

${ }^{\mathrm{c}}$ Compounds in trace amounts and hidden by other. Their UV spectra have not been observed properly.

Table 2

$R_{\mathrm{t}}, \mathrm{UV},-\mathrm{MS}:[\mathrm{M}-\mathrm{H}]^{-},-\mathrm{MS}^{2}[\mathrm{M}-\mathrm{H}]^{-}$and $-\mathrm{MS}^{3}[(\mathrm{M}-\mathrm{H}) \rightarrow(\mathrm{M}-\mathrm{H}-162)]^{-}$data of flavonol acyl-glycosides found in $P$. brassicae ${ }^{\mathrm{a}}$

\begin{tabular}{|c|c|c|c|c|c|c|c|c|c|}
\hline \multirow[t]{3}{*}{ Compounds $^{\mathrm{b}}$} & \multirow{3}{*}{$\begin{array}{l}R_{\mathrm{t}} \\
(\min )\end{array}$} & \multirow[t]{3}{*}{$\mathrm{UV}(\mathrm{nm})$} & \multirow{3}{*}{$\begin{array}{l}{[\mathrm{M}-\mathrm{H}]^{-}} \\
(\mathrm{m} / \mathrm{z})\end{array}$} & \multicolumn{6}{|c|}{ Flavonol-3(acyl)glycosyl-7-glucoside derivatives } \\
\hline & & & & \multirow{2}{*}{$\frac{\mathrm{MS}^{2}[\mathrm{M}-\mathrm{H}]^{-}}{\text {-Glc }(162)}$} & \multicolumn{5}{|c|}{$\mathrm{MS}^{3}[(\mathrm{M}-\mathrm{H}) \rightarrow(\mathrm{M}-\mathrm{H}-162)]^{-}$} \\
\hline & & & & & $\begin{array}{l}-\mathrm{Caf} \\
(162)\end{array}$ & $\begin{array}{l}-\mathrm{MCaf} \\
(192)\end{array}$ & $\begin{array}{l}-p \text { Coum } \\
(146)\end{array}$ & $\begin{array}{l}\text {-Fer } \\
(176)\end{array}$ & $\begin{array}{l}\text {-Sinp } \\
(206)\end{array}$ \\
\hline 4 Querc-3-(Fer)triGlc-7-Glc & 10.1 & $\begin{array}{l}265,291 \mathrm{sh}, \\
321\end{array}$ & 1125 & 963 & & & & 787 & \\
\hline 5 Kaempf-3-(Sinp)triGlc-7-Glc & 11.1 & $\begin{array}{l}269,290 \mathrm{sh}, \\
325\end{array}$ & 1139 & 977 & & & & & 771 \\
\hline 6 Kaempf-3-(Fer)triGlc-7-Glc & 11.8 & $\begin{array}{l}270,299 \mathrm{sh}, \\
323\end{array}$ & 1109 & 947 & & & & 771 & \\
\hline 7 Kaempf-3-(p-Coum)triGlc-7-Glc ${ }^{c}$ & 12.5 & & 1079 & 917 & & & 771 & & \\
\hline 8 Kaempf-3-(MCaf)Soph-7-Glc & 13.6 & 271,325 & 963 & 801 & & 609 & & & \\
\hline 9 Kaempf-3-(Caf)Soph-7-Glc & 14.8 & 269,320 & 933 & 771 & 609 & & & & \\
\hline 17 Kaempf-3-(diSinp)triGlc-7-Glc ${ }^{\mathrm{d}}$ & 27.2 & 270,331 & 1345 & 1183 & & & & & $977^{\mathrm{d}}$ \\
\hline \multirow[t]{4}{*}{18 Kaempf-3-(FerSinp)triGlc-7-Gle ${ }^{\mathrm{e}}$} & 28.6 & 271,331 & 1315 & 1153 & & & & 977 & $947^{\mathrm{e}}$ \\
\hline & & & & \multicolumn{6}{|c|}{ Flavonol-3(acyl)glycoside derivatives } \\
\hline & & & & & \multicolumn{5}{|c|}{$\mathrm{MS}^{2}[\mathrm{M}-\mathrm{H}]^{-}$} \\
\hline & & & & & $-\mathrm{Caf}$ & $-\mathrm{MCaf}$ & $-p$ Coum & -Fer & -Sinp \\
\hline 10 Querc-3-(p-Coum)Soph ${ }^{\mathrm{c}}$ & 15.8 & & 771 & & & & 625 & & \\
\hline 11 Kaempf-3-( $p$-Coum)triGlc & 16.2 & $\begin{array}{l}270 \mathrm{sh}, 293 \mathrm{sh}, \\
315\end{array}$ & 917 & & & & 771 & & \\
\hline 12 Kaempf-3-(p-Coum)Soph ${ }^{\mathrm{c}}$ & 18.2 & & 755 & & & & 609 & & \\
\hline 13 Kaempf-3-(MCaf)Soph & 18.9 & 269,325 & 801 & & & 609 & & & \\
\hline 16 Kaempf-3-(p-Coum)Soph (isom) & 26.1 & 267,338 & 755 & & & & 609 & & \\
\hline 19 Querc-3-(Fer)triGlc & 29.5 & 269,327 & 963 & & & & & 787 & \\
\hline
\end{tabular}

${ }^{a}$ Main observed fragments. Other ions were found but they have not been included.

b Querc, quercetin; Kaempf, kaempferol; Soph, sophoroside; Glc, glucoside; Fer, feruloyl; Sinp, sinpapoyl; p-Coum, p-coumaroyl; Caf, caffeoyl; MCaf, methoxycaffeoyl.

${ }^{\mathrm{c}}$ Compounds in trace amounts and hidden by other. Their UV spectra have not been observed properly.

${ }^{d} \operatorname{MS}^{4}(1345 \rightarrow 1183 \rightarrow 977): 771$.

${ }^{\mathrm{e}} \mathrm{MS}^{4}(1315 \rightarrow 1153 \rightarrow 947): 771$.

kaempferol and quercetin derivatives, the later in lesser amounts, with a high degree of glycosilation, as well as a possible acylation at the glycosidic fraction linked to the hydroxyl group at the 3 position of the flavonol.

From the MS study it is possible to distinguish a group of flavonol glycoside derivatives without acylation (Table 1 ), whose MS fragmentations (Ferreres et al., 2004) are characteristic of flavonol-3- $O$-sophoroside-7- $O$-glucoside (compounds 1 and 2) (Fig. 2), flavonol-3-O-sophoroside7-O-sophoroside (3), flavonol-3-O-sophoroside (14 and 15) and flavonol-3- $O$-glucoside (20).
On the other hand, the MS fragmentation pattern of the acylated flavonol derivatives (Vallejo et al., 2004) confirms the presence of a series of flavonol-3-O-(acyl)glycoside-7-O-glucoside (Table 2) (compounds 4-9, 17 and 18), in which we can observe a loss of $162 \mathrm{u}$ in the $\mathrm{MS}^{2}[\mathrm{M}-\mathrm{H}]^{-}$due to the break of glucose at the 7-position. The $\mathrm{MS}^{3}[(\mathrm{M}-\mathrm{H}) \rightarrow(\mathrm{M}-\mathrm{H}-162)]^{-}$shows the loss of the acyl radical giving the aglycone fragment linked to the glycosidic fraction at the 3-position, triglucoside (4-7) or sophorose (8 and 9) (Table 2). For the diacylated derivatives, compounds $\mathbf{1 7}$ and 18, a new MS event 


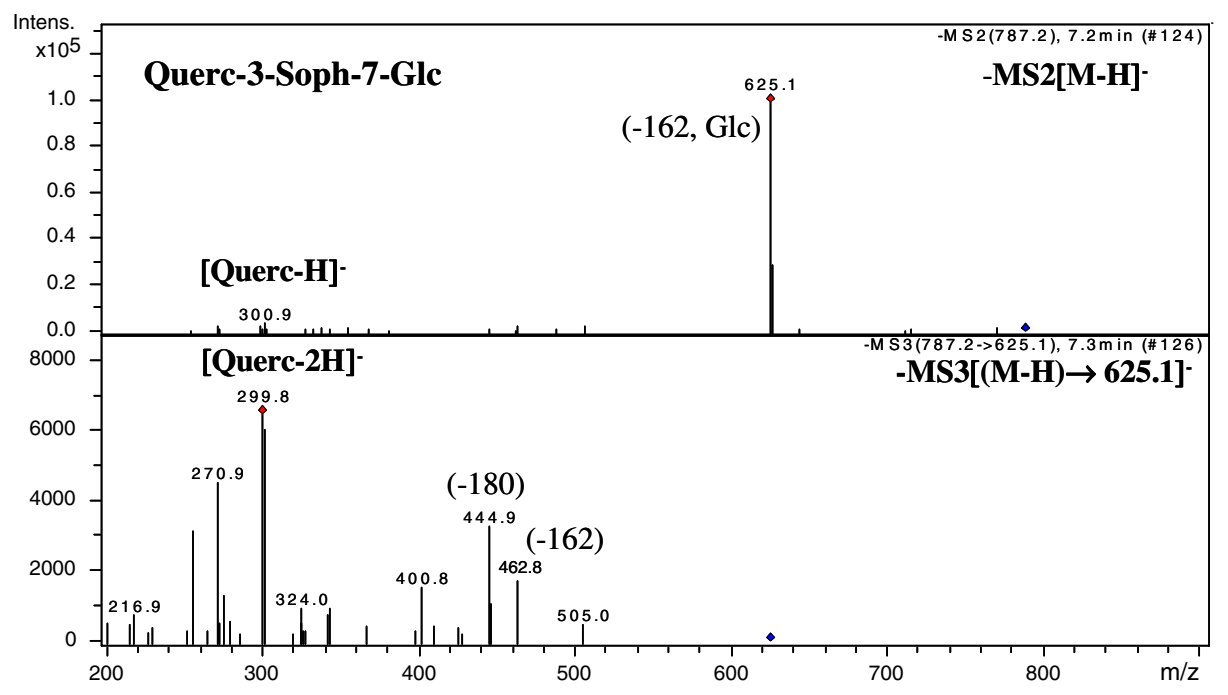

Fig. 2. $\mathrm{MS}^{n}$ analysis of quercetin 3-O-sophoroside-7-O-glucoside (1).

$\left(\mathrm{MS}^{3}[(\mathrm{M}-\mathrm{H}) \rightarrow(\mathrm{M}-\mathrm{H}-162) \rightarrow(\mathrm{M}-\mathrm{H}-162 \text {-acyl })]^{-}\right) \quad$ was necessary to obtain the referred fragment.

Another set of complex flavonol derivatives (compounds 10-13, 16 and 19) shows a MS fragmentation (Table 2) in which $\mathrm{MS}^{2}[\mathrm{M}-\mathrm{H}]^{-}$event the loss could be interpreted as that of an acyl radical to give rise to the aglycone fragment linked to the glycosidic fraction at the 3-position (Figs. 3 and 4), confirming the lack of glycosilation at the 7-position (Vallejo et al., 2004). In this group of compounds it is observed an apparently anomalous chromatographic behaviour of compounds 10,12 and $\mathbf{1 3}$, once they elute before the deacylated compounds from which they derive from. This behaviour has already been described for similar compounds found in cauliflower (Llorach et al., 2003), being noticed that the acylated derivatives at the 3-position of the sugar and without glycosilation in the 7-position exhibited an apparent irregular retention time. On the other hand, the presence of two isomers of kaempferol 3-O-( $p$ coumaroyl)-sophoroside with lower (compound 12) and higher retention times (compound 16) than kaempferol 3$O$-sophoroside (15) indicates that the position of the acylation over the sugar also influences the order of elution.

In addition, the loss of $146 \mathrm{u}$ in the MS fragmentation of compounds 7, 10, 11, 12 and 16, the majority of them with poor or badly defined UV spectrum as mentioned above, could be due to the presence of a rhamnosyl radical, instead of a $p$-coumaroyl one. However, in tronchuda cabbage leaves neither rhamnosyl nor $p$-coumaroyl derivatives were found (Ferreres et al., 2005, 2006). So, we consider that the demethoxylation of the sinapoyl and/or feruloyl derivatives originating $p$-coumaroyl derivatives is more likely to occur during the metabolism process in the larvae, as indicated below, rather than the demethylation of glucose to give rise to rhamnosyl derivatives.

Thus, in $P$. brassicae the following compounds were identified or tentatively identified: quercetin 3-O-sophoroside-7$O$-glucoside (1), kaempferol 3-O-sophoroside-7- $O$-glucoside (2), kaempferol 3- $O$-sophoroside-7- $O$-sophoroside (3), querce-

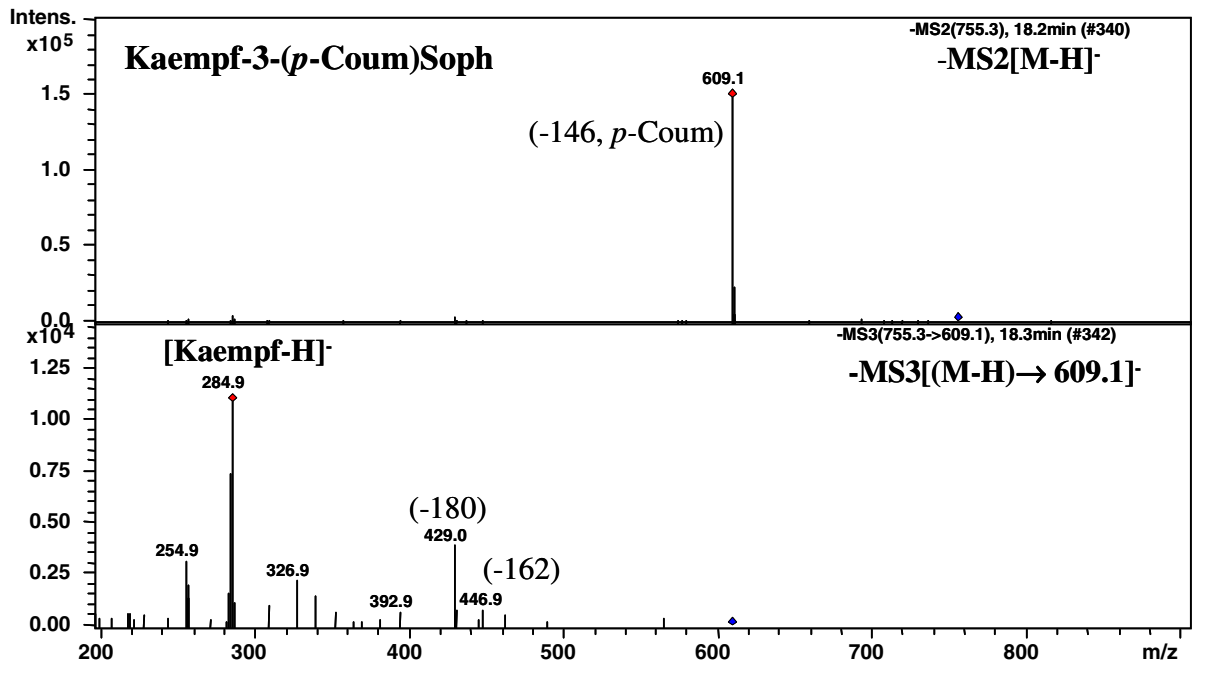

Fig. 3. $\mathrm{MS}^{n}$ analysis of kaempferol 3-O-(p-coumaroyl)-sophoroside (12). 


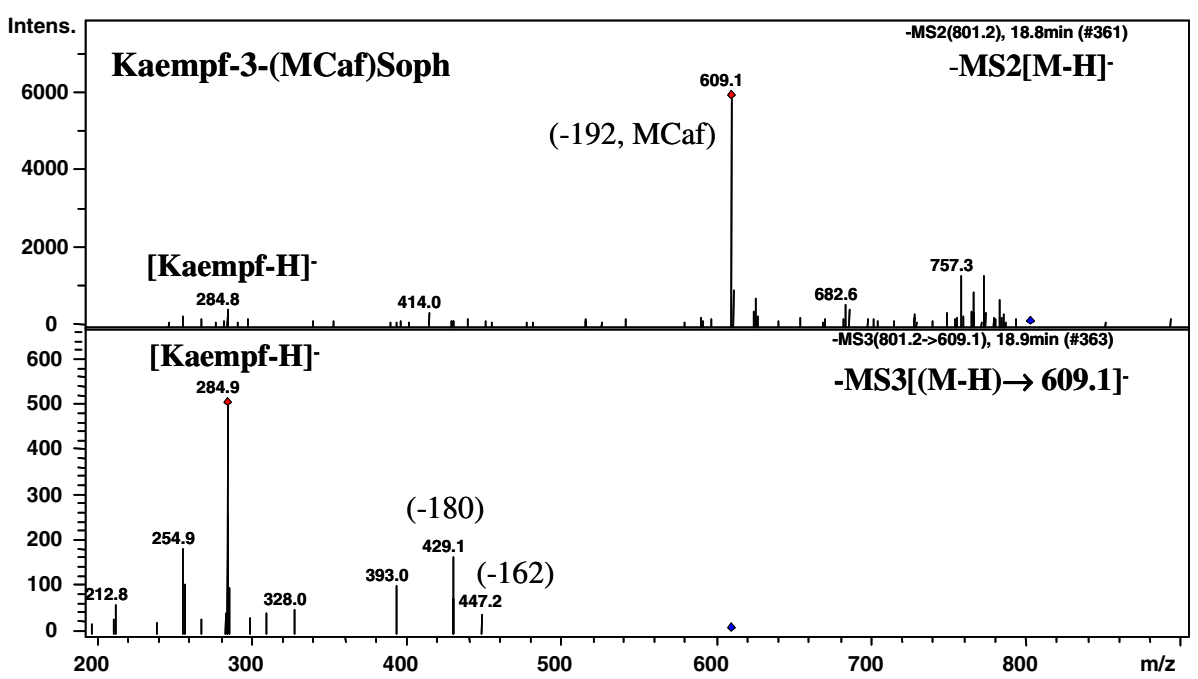

Fig. 4. $\mathrm{MS}^{n}$ analysis of kaempferol 3-O-(methoxycaffeoyl)-sophoroside (13).

tin 3-O-(feruloyl)-triglucoside-7- $O$-glucoside (4), kaempferol 3-O-(sinapoyl)-triglucoside-7- $O$-glucoside (5), kaempferol 3$O$-(feruloyl)-triglucoside-7-O-glucoside (6), kaempferol 3-O-( $p$-coumaroyl)-triglucoside-7- $O$-glucoside (7) (tentatively), kaempferol 3-O-(methoxycaffeoyl)-sophoroside-7$O$-glucoside (8) (tentatively), kaempferol 3-O-(caffeoyl)sophoroside-7-O-glucoside (9) (tentatively), quercetin 3-O-( $p$-coumaroyl)-sophoroside (10) (tentatively), kaempferol 3-O-( $p$-coumaroyl)-triglucoside (11) (tentatively), two isomers of kaempferol 3-O-( $p$-coumaroyl)-sophoroside (12 and 16) (tentatively), kaempferol 3-O-(methoxycaffeoyl)sophoroside (13) (tentatively), quercetin 3-O-sophoroside (14), kaempferol 3- $O$-sophoroside (15), kaempferol 3- $O$-(disinapoyl)-triglucoside-7- $O$-glucoside (17), kaempferol 3-O(feruloyl/sinapoyl)-triglucoside-7-O-glucoside (18), quercetin 3- $O$-(feruloyl)-triglucoside (19) and kaempferol 3-O-glucoside (20).

\subsection{Comparison between flavonoids in P. brassicae and tronchuda cabbage host}

The phenolic composition of the tronchuda cabbage external leaves is well defined in a previous work, analysing several samples, from both organic and conventional agricultural practices (Ferreres et al., 2005). The composition of the host leaves, from which the larvae feed, revealed to be similar to that described before, being detected 13 kaempferol derivatives (Table 3). The flavonoid profile obtained with $P$. brassicae was then compared with that of the cabbage. Kaempferol 3-O-sophoroside-7-O-glucoside, kaempferol 3-O-sophoroside-7- $O$-sophoroside and kaempferol 3-O-sophoroside were the only compounds that the larvae and cabbage had in common.

Although the glycosylation pattern of the flavonols is the same in both extracts, it can be observed that the flavonol 3-O-glycosides represent more than ca. $50 \%$ in the larvae extract (Table 4), while they correspond to ca. $12 \%$ of their food plant (Table 3). This can be ascribed to the
Table 3

Phenolic composition of tronchuda cabbage external leaves

\begin{tabular}{lr}
\hline Compound & $\%$ \\
\hline 21+ Kaempferol 3- $O$-sophorotrioside-7- $O$-glucoside & 7.6 \\
22 Kaempferol 3- $O$-(methoxycaffeoyl/caffeoyl)-sophoroside-7- $O$ - & \\
$\quad$ glucoside & 22.9 \\
2 Kaempferol 3- $O$-sophoroside-7- $O$-glucoside & 1.4 \\
23 Kaempferol 3- $O$-sophorotrioside-7- $O$-sophoroside & 11.4 \\
3+ Kaempferol 3- $O$-sophoroside-7- $O$-sophoroside & \\
24 Kaempferol 3- $O$-tetraglucoside-7- $O$-sophoroside & 17.1 \\
25 Kaempferol 3- $O$-(sinapoyl/caffeoyl)-sophoroside-7- $O$-glucoside & 27.8 \\
26 Kaempferol 3- $O$-(feruloyl/caffeoyl)-sophoroside-7- $O$-glucoside & 5.1 \\
27+ Kaempferol 3- $O$-sophorotrioside & \\
28 Kaempferol 3- $O$-(sinapoyl)-sophoroside & 0.4 \\
29 Kaempferol 3- $O$-(feruloyl)-sophorotrioside & 1.1 \\
30 Kaempferol 3- $O$-(feruloyl)-sophoroside & 5.2 \\
15 Kaempferol 3- $O$-sophoroside &
\end{tabular}

Table 4

Phenolic composition of P. brassicae

\begin{tabular}{lr}
\hline Compound & $\%$ \\
\hline 1 Quercetin 3- $O$-sophoroside-7- $O$-glucoside & 8.7 \\
2 Kaempferol 3- $O$-sophoroside-7- $O$-glucoside & 10.0 \\
3 Kaempferol 3- $O$-sophoroside-7- $O$-sophoroside & 6.6 \\
4 Quercetin 3- $O$-(feruloyl)-triglucoside-7- $O$-glucoside & 4.5 \\
5 Kaempferol 3- $O$-(sinapoyl)-triglucoside-7- $O$-glucoside & 5.0 \\
6 Kaempferol 3- $O$-(feruloyl)-triglucoside-7- $O$-glucoside & 5.6 \\
7 Kaempferol 3- $O$-( $p$-coumaroyl)-triglucoside-7- $O$-glucoside & 2.6 \\
8 Kaempferol 3- $O$-(methoxycaffeoyl)-sophoroside-7- $O$-glucoside & 0.5 \\
9 Kaempferol 3- $O$-(caffeoyl)-sophoroside-7- $O$-glucoside & 1.8 \\
10 Quercetin 3- $O$-( $p$-coumaroyl)-sophoroside & 3.4 \\
11 Kaempferol 3- $O$-(p-coumaroyl)-triglucoside & 3.3 \\
12 Kaempferol 3- $O$-( $(p$-coumaroyl)-sophoroside & 13.4 \\
13+ Kaempferol 3- $O$-(methoxycaffeoyl)-sophoroside & 9.2 \\
14 Quercetin 3- $O$-sophoroside & \\
15 Kaempferol 3- $O$-sophoroside & 15.8 \\
16 Kaempferol 3- $O$-( $p$-coumaroyl)-sophoroside (isomer) & 2.4 \\
17 Kaempferol 3- $O$-(disinapoyl)-triglucoside-7- $O$-glucoside & 2.1 \\
18 Kaempferol 3- $O$-(feruloyl/sinapoyl)-triglucoside-7- $O$-glucoside & 1.3 \\
19 Quercetin 3- $O$-(feruloyl)-triglucoside & 1.9 \\
20 Kaempferol 3- $O$-glucoside & 1.9 \\
\hline
\end{tabular}


metabolism of the flavonols glycosylated at 3 and 7 positions present on tronchuda cabbage, or to a higher efficiency of sequestration of flavonol 3-O-glycosides.

Among the flavonol glycoside derivatives without acylation, kaempferol 3-O-sophoroside is the most abundant, corresponding to ca. $16 \%$ of the total amount of phenolic compounds of the larvae (Table 4), while in tronchuda cabbage leaves it only represented ca. 5\% (Table 3). This difference possibly results from the metabolism of kaempferol 3-O-sophoroside-7-O-glucoside and its acylated derivatives, which are the most abundant compounds of tronchuda cabbage external leaves.

In what concerns the presence of quercetin derivatives, the composition of the external leaves of tronchuda cabbage (Ferreres et al., 2005) was re-analysed and these were detected in vestigial amounts, which also happened with the tronchuda cabbage external leaves eaten by P. brassicae. The larvae contain high amounts of quercetin derivatives (ca. $18 \%$ of the total amount of phenolic compounds) (Table 4), while in tronchuda cabbage these compounds are present only in trace amounts, suggesting that $P$. brassicae selectively sequester these flavonoids or that the kaempferol glycosides are metabolised into quercetin glycosides by the larvae.

The presence of $p$-coumaroyl derivatives, which have not been found on either the internal or external leaves of tronchuda cabbage (Ferreres et al., 2005; Sousa et al., 2005) can be explained by the demethoxylation of the sinapoyl and/or feruloyl derivatives during the metabolism process in the larvae. On the other hand, the absorbance of the peaks observed in Fig. 1 for acylated flavonoid derivatives, cannot be taken as proportional to their abundance, as some of them co-eluted with other unidentified cinnamoyl acids derivatives, presenting a similar UV spectrum and contributing to the overall absorbance of those peaks. Another possible explanation is their existence in tronchuda cabbage leaves in concentrations below the detection limits, being, however, selectively uptaken and accumulated by $P$. brassicae.

The existence of two methoxylated flavonol derivatives in P. brassicae (compounds 8 and 13) (Fig. 4) can be resultant from the metabolism of kaempferol 3-O-(methoxycaffeoyl/caffeoyl)-sophoroside-7- $O$-glucoside present on the external leaves of the tronchuda cabbage.

As far as we know, this is the first report about the uptake of flavonoids by $P$. brassicae and it was observed they sequestered flavonoids from tronchuda cabbage external leaves. In addition, some of them may undergo metabolism during ingestion. The results suggest that $P$. brassicae may have interest for the synthesis and/or accumulation of potential health promoting compounds, which are rather unusual in nature. Although the P. brassicae gut was not removed and longer starving periods should be tested, some conclusions could be obtained as larvae and feeding plant contain only three compounds in common, indicating that the 17 different phenolics found in larvae are directly related with the insect, being a consequence of its metabo- lism and/or selective sequestration. Further studies should be done, varying the starving periods before freezing and analysing gut and remaining body separately, in order to obtain more information about the metabolic process. Larvae in different instars should also be studied, to evaluate the effect of the developmental stage in sequestration rate of flavonoids. Also the study of the faeces of larvae can provide valuable data about the excretion of flavonoids.

\section{Experimental}

\subsection{Standards and reagents}

Kaempferol 3-O-rutinoside, kaempferol 3-O-glucoside and quercetin 3-O-rutinoside were from Extrasynthése (Genay, France). Methanol, formic and acetic acid were purchased from Merck (Darmstadt, Germany). The water was treated in a Milli-Q water purification system (Millipore, Bedford, MA).

\subsection{Larvae, plant material and sampling}

Wild P. brassicae larvae (fourth instar) and respective tronchuda cabbage external leaves (of three individuals with 45 days-old) host plants were collected on fields located in Samil, Bragança, northeastern Portugal. Voucher specimens of tronchuda cabbage leaves are deposited at Serviço de Farmacognosia from Faculdade de Farmácia, Universidade do Porto. After collection the larvae were kept without food for $1 \mathrm{~h}$ before they were frozen. The frozen larvae and plant material were freeze-dried and kept in a dessicator until analysis.

\subsection{Extraction of the phenolic compounds}

The identification of the phenolic compounds was performed using a hydromethanolic extract of the lyophilised larvae: ca. $1.5 \mathrm{~g}$ powdered larvae was thoroughly mixed with $2.5 \mathrm{ml}$ methanol-water (1:1), ultra-sonicated and filtered.

The same extraction methodology was used for quantification purposes of phenolic compounds in larvae and plant material.

\subsection{HPLC-DAD-MS/MS-ESI qualitative analysis}

Chromatographic separations were carried out on a $250 \mathrm{~mm} \times 4 \mathrm{~mm}, 5$ - $\mu \mathrm{m}$ particle size, RP-18 LiChroCART (Merck, Darmstadt, Germany) column protected with a $4 \mathrm{~mm} \times 4 \mathrm{~mm}$ LiChroCART guard column using acetic acid $1 \%(\mathrm{~A})$ and methanol (B) as solvents, starting with $20 \% \mathrm{~B}$ and using a gradient to obtain $50 \%$ B at $35 \mathrm{~min}$. The flow rate was $1 \mathrm{ml} \mathrm{min}{ }^{-1}$ and the injection volume was $20 \mu 1$. The HPLC system was equipped with an Agilent 1100 Series diode array and a mass detector in series (Agilent Technologies, Waldbronn, Germany). It consisted of a G1312A binary pump, an G1313A autosampler, a G1322A 
degasser and a G1315B photodiode array detector controlled by a ChemStation software (Agilent, v. 08.03). Spectroscopic data from all peaks were accumulated in the range $240-400 \mathrm{~nm}$, and chromatograms were recorded at $330 \mathrm{~nm}$. The mass detector was a G2445A Ion-Trap Mass Spectrometer equipped with an electrospray ionisation (ESI) system and controlled by LCMSD software (Agilent, v. 4.1.). Nitrogen was used as nebulising gas at a pressure of $65 \mathrm{psi}$ and the flow was adjusted to $111 \mathrm{~min}^{-1}$. The heated capillary and voltage were maintained at $350^{\circ} \mathrm{C}$ and $4 \mathrm{kV}$, respectively. The full-scan mass covered the range from $\mathrm{m} / \mathrm{z} 90$ up to $\mathrm{m} / \mathrm{z} 2000$. Collisioninduced fragmentation experiments were performed in the ion trap using helium as collision gas, with voltage ramping cycles from 0.3 up to $2 \mathrm{~V}$. MS data were acquired in the negative ionisation mode. $\mathrm{MS}^{n}$ data were achieved in the automatic mode on the more abundant fragment ion in $\mathrm{MS}^{n-1}$. Tables 1 and 2 show the most frequent ions which characterise the fragmentation of the compounds. Other ions were found but they have not been included due to their low significance on the MS behaviour ions.

\subsection{HPLC-DAD quantitative analysis}

Twenty microliters of each extract were analysed using a HPLC unit (Gilson) and a $250 \times 4.6 \mathrm{~mm}$ i.d., $5 \mu \mathrm{m}$ Spherisorb ODS2 column (Waters, Milford, USA). The solvent system was a mixture of formic acid $5 \%$ in water (A) and methanol (B), with a flow rate of $1 \mathrm{ml} \mathrm{min}{ }^{-1}$, and the gradient was as follows: $0 \mathrm{~min}-10 \% \mathrm{~B} ; 25 \mathrm{~min}-20 \% \mathrm{~B} ; 40 \mathrm{~min}-$ $50 \%$ B; $45 \min -50 \%$ B; $46 \min -90 \%$ B; $50 \min -90 \%$ B; $55 \min -100 \% \mathrm{~B} ; 58 \mathrm{~min}-100 \% \mathrm{~B}$; and $60 \min -10 \% \mathrm{~B}$. Detection was achieved with a Gilson diode array detector. Spectroscopic data from all peaks were accumulated in the range of 200-400 nm, and chromatograms were recorded at $330 \mathrm{~nm}$. The data were processed on Unipoint system Software (Gilson Medical Electronics, Villiers le Bel, France). Peak purity was checked by the software contrast facilities.

Phenolic compounds quantification was achieved by the peak areas recorded in the chromatograms relative to that registered for known concentrations of external standards. With the exception of kaempferol 3-O-glucoside, which was quantified as it, the other kaempferol and quercetin derivatives were quantified as kaempferol 3-O-rutinoside and quercetin 3-O-rutinoside, respectively, since none of the identified compounds was commercially available.

\section{Acknowledgements}

The authors are grateful to Fundação para a Ciência e Tecnologia (PTDC/AGR-AAM/64150/2006) for financial support of this work.

\section{References}

Burghardt, F., Fiedler, K., Proksch, P., 1997. Uptake of flavonoids from Vicia villosa (Fabaceae) by the lycaenid butterfly, Polyommatus icarus (Lepidoptera: Lycaenidae). Biochem. Syst. Ecol. 25, 527-536.

Burghardt, F., Knüttel, H., Becker, M., Fiedler, K., 2000. Flavonoid wing pigments increase attractiveness of female common blue (Polyommatus icarus) butterflies to mate-searching males. Naturwissenschaften 87 , 304-307.

Burghardt, F., Proksch, P., Fiedler, K., 2001. Flavonoid sequestration by the common blue butterfly Polyommatus icarus: quantitative intraspecific variation in relation to larval hostplant, sex and body size. Biochem. Syst. Ecol. 29, 875-889.

Ferreres, F., Llorach, R., Gil-Izquierdo, A., 2004. Characterization of the interglycosidic linkage in di-, tri-, tetra- and pentaglycosylated flavonoids and differentiation of positional isomers by liquid chromatography/electrospray ionization tandem mass spectrometry. J. Mass Spectrom. 39, 312-321.

Ferreres, F., Valentão, P., Llorach, R., Pinheiro, C., Cardoso, L., Pereira, J.A., Sousa, C., Seabra, R.M., Andrade, P.B., 2005. Phenolic compounds in external leaves of tronchuda cabbage (Brassica oleracea L. var. costata DC). J. Agric. Food Chem. 53, 2901-2907.

Ferreres, F., Sousa, C., Vrchovská, V., Valentão, P., Pereira, J.A., Seabra, R.M., Andrade, P.B., 2006. Chemical composition and antioxidant activity of tronchuda cabbage internal leaves. Eur. Food Res. Technol. 222, 88-98

Geuder, M., Wray, V., Fiedler, K., Proksch, P., 1997. Sequestration and metabolism of host-plant flavonóides by the lycaenid butterfly Polyommatus bellargus. J. Chem. Ecol. 23, 1361-1372.

Harborne, J.B., Grayer, R.J., 1994. Flavonoids and insects. In: Harborne, J.B. (Ed.), The Flavonoids - Advances in Research Since 1986. Chapman \& Hall, London, pp. 589-618.

Knüttel, H., Fiedler, K., 2001. Host-plant-derived variation in ultraviolet wing patterns influences mate selection by male butterflies. J. Exp. Biol. 204, 2447-2459.

Llorach, R., Gil-Izquierdo, A., Ferreres, F., Tomás-Barberán, F.A., 2003. HPLC-DAD-MS/MS ESI characterization of unusual highly glycosylated acylated flavonoids from cauliflower (Brassica oleracea L. var. botrytis) agroindustrial byproducts. J. Agric. Food Chem. 51, 38953899.

Renwick, J.A.A., 2002. The chemical world of crucivores: lures, treats and traps. Entomol. Exp. Appl. 104, 35-42.

Schittko, U., Burghardt, F., Fiedler, K., Wray, V., Proksch, P., 1999. Sequestration and distribution of flavonoids in the common blue butterfly Polyommatus icarus reared on Trifolium repens. Phytochemistry $51,609-614$.

Sousa, C., Valentão, P., Rangel, J., Lopes, G., Pereira, J.A., Ferreres, F., Seabra, R.M., Andrade, P.B., 2005. Influence of two fertilization regimens on the amounts of organic acids and phenolic compounds of tronchuda cabbage (Brassica oleracea L. var. costata DC). J. Agric. Food Chem. 53, 9128-9132.

Vallejo, F., Tomás-Barberán, F.A., Ferreres, F., 2004. Characterisation of flavonols in broccoli (Brassica oleracea L. var. italica) by liquid chromatography-UV diode-array detection-electrospray ionisation mass spectrometry. J. Chromatogr. A 1054, 181-193.

van Loon, J.J.A., Wang, C.Z., Nielsen, J.K., Gols, R., Qiu, Y.T., 2002. Flavonoids from cabbage are feeding stimulants for diamondback moth larvae additional to glucosinolates: chemoreception and behaviour. Entomol. Exp. Appl. 104, 27-34.

Vrchovská, V., Sousa, C., Valentão, P., Ferreres, F., Pereira, J.A., Seabra, R.M., Andrade, P.B., 2006. Antioxidative properties of tronchuda cabbage (Brassica oleracea L. var. costata DC) external leaves against DPPH, superoxide radical, hydroxyl radical and hypochlorous acid. Food Chem. 98, 416-425. 